\title{
Parametric Analysis of Life Support Systems for Future Space Exploration Missions
}

\author{
Michael J. Swickrath* and Molly S. Anderson ${ }^{\dagger}$ and (Additional Authors) \\ NASA Johnson Space Center, Houston, TX, r7058 \\ Bob M. Bagdigian $\ddagger$ \\ NASA Marshall Space Flight Center, Huntsville, AL, 35812
}

\begin{abstract}
Having adopted a flexible path approach to space exploration, the National Aeronautics and Space Administration is in a process of evaluating future targets for space exploration. In order to maintain the welfare of a crew during future missions, a suite of life support technology is responsible for oxygen and water generation, carbon dioxide control, the removal of trace concentrations of organic contaminants, processing and recovery of water, and the storage and reclamation of solid waste. For each particular life support subsystem, a variety competing technologies either exist or are under aggressive development efforts. Each individual technology has strengths and weaknesses with regard to launch mass, power and cooling requirements, volume of hardware and consumables, and crew time requirements for operation. However, from a system level perspective, the favorability of each life support architecture is better assessed when the sub-system technologies are analyzed in aggregate. In order to evaluate each specific life support system architecture, the measure of equivalent system mass (ESM) was employed to benchmark system favorability. Moreover, the results discussed herein will be from the context of loop-closure with respect to the air, water, and waste sub-systems. Specifically, closure relates to the amount of consumables mass that crosses the boundary of the vehicle over the lifetime of a mission. As will be demonstrated in this manuscript, the optimal level of loop closure is heavily dependent upon mission requirements such as duration and the level of extravehicular activity (EVA) performed. Sub-system level trades were also considered as a function of mission duration to assess when increased loop closure is practical. Although many additional factors will likely merit consideration in designing life support systems for future missions, the ESM results described herein provide a context for future architecture design decisions toward a flexible path program.
\end{abstract}

*Analyst, Crew and Thermal Systems Division, 2101 NASA Parkway/EC211, Houston, TX, 77058, AIAA Member.

${ }^{\dagger}$ Analysis Lead, Crew and Thermal Systems Division, 2101 NASA Parkway/EC211, Houston, TX, 77058, AIAA Member.

${ }^{\ddagger}$ Chief, Environmental Control and Life Support Development Branch, Marshall Space Flight Center/ES62, Huntsville, AL, 35812, Member AIAA. 